\title{
Comment on: 'Immediate IOP elevation after transscleral cyclophotocoagulation'
}

\author{
Lorraine Ochieng ${ }^{1} \cdot$ Nuwan Niyadurupola $^{2} \cdot$ David Broadway $^{2} \cdot$ Tom Eke $^{2}$
}

Received: 26 November 2017 / Accepted: 2 January 2018 / Published online: 23 February 2018

(c) The Royal College of Ophthalmologists 2018

We were concerned by Razeghinejad et al's recent publication on intraocular pressure (IOP) immediately following trans-scleral cyclophotocoagulation (TSCPC) or cyclodiode laser treatment [1]. They found a worryingly high rate of significant IOP rise (IOP spike), with $90 \%$ of their patients receiving intravenous mannitol. Therefore, we audited our own practice.

This prospective audit was approved by our local Clinical Audit Department. Our default protocol is 40 shots at $1500 \mathrm{~mW}, 1500 \mathrm{~ms}$, using the G-probe [2]. Power is reduced if there are repeated 'pops'. Additional IOPlowering medications are not normally given. For the audit, we measured IOP before the anaesthetic, after anaesthetic, and immediately after TSCPC, with the patient in the same position. We used the Tonopen AVIA
(Reichert, NY, USA) and averaged three 'reliable' IOP's for each time-point. Any IOP rise that we considered 'potentially clinically significant' was managed according to clinical judgement.

We audited 35 consecutive procedures in 5 months. The only exclusions were eyes which could not have IOP measured by Tonopen (one un-cooperative patient, a few with very-high initial IOP). All had local anaesthesia (LA), either peribulbar or sub-Tenon's. 'Pops' occurred in six eyes (17\%). There was a low rate of IOP spikes (Fig. 1). There was only one 'potentially clinically significant' IOP rise, and this resolved without active intervention. The IOP was 27 $\mathrm{mmHg}$ before LA, $23 \mathrm{mmHg}$ after LA, and $35 \mathrm{mmHg}$ after TSCPC (40 shots at $1500 \mathrm{~mW}, 1500 \mathrm{~ms}$; three 'pops'). The patient was simply observed; 20 minutes later the IOP was
Fig. 1 The intra-ocular pressure (IOP) profile after cyclodiode laser treatment. We used our standard protocol of 40 shots at $1500 \mathrm{~mW}, 1500 \mathrm{~ms}$. Two patients had an IOP rise that was considered 'potentially clinically significant' (red lines), though both resolved without medication. See text for further detail

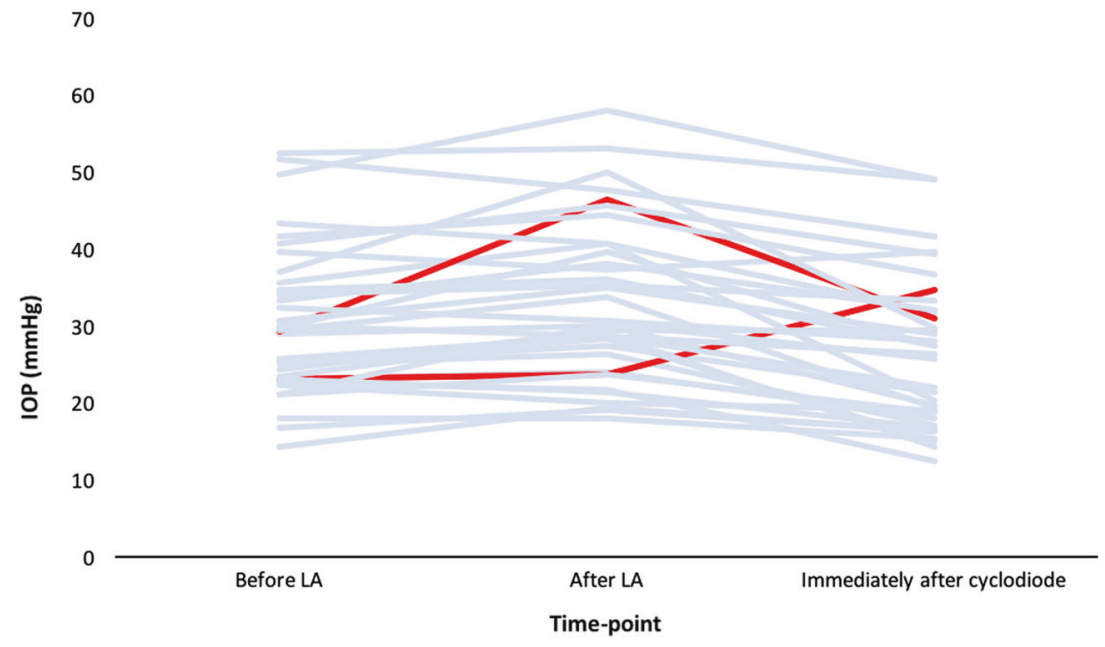

Tom Eke

tom.eke@nnuh.nhs.uk

1 University of East Anglia, Norwich NR4 7TJ, UK

2 Norfolk and Norwich University Hospital, Colney Lane, Norwich NR4 7UY, UK 
$32 \mathrm{mmHg}$. No additional IOP-lowering medication was given, IOP was $4 \mathrm{mmHg}$ one week later. Another patient had an IOP rise following peribulbar LA; this resolved with massage.

With our technique, we were reassured by the absence of sight-threatening IOP spikes. We attribute this to the low power settings: 40 shots at $1500 \mathrm{~mW}, 1500 \mathrm{~ms}$, with reduced power if pops are heard. By contrast, Razeghinejad's team used higher initial power $(2000 \mathrm{~mW}, 2000 \mathrm{~ms})$, and seemed to increase the power until they heard 'pops' (maximum $3000 \mathrm{~mW}, 4000 \mathrm{~ms}$ ). Razeghinejad speculated that the 'pops' probably cause IOP spikes, due to intraocular gas bubbles. Our audit indicates that the lower-power protocol and minimising 'pops' is preferable for TSCPC.

\section{Compliance with ethical standards}

Conflict of interest The authors declare that they have no conflict of interest.

\section{References}

1. Razeghinejad MR, Hamid A, Nowroozzadeh MH. Immediate IOP elevation after transscleral cyclophotocoagulation. Eye (Lond). 2017;31:1249-50.

2. Tsatsos M, Burnett CA, Broadway DC, Eke T. Local anaesthesia for trans-scleral cyclodiode laser procedures: surgeon and patient satisfaction with sub-Tenon's and peribulbar anaesthesia. Clin Exp Ophthalmol. 2011;39:472. 